\title{
Inhaled nitric oxide treatment for preterm infants with hypoxic respiratory failure
}

\author{
Rosalind L Smyth \\ Institute of Child Health, Alder Hey Children's Hospital, Liverpool L12 2AP, UK
}

Introductory articles

\section{Inhaled nitric oxide in premature neonates with severe hypoxaemic respiratory failure: a randomised controlled trial}

\author{
JP Kinsella, WF Walsh, CL Bose, DR Gerstmann, JJ Labella, S Sardesai, MC Walsh-Sukys, \\ MJ McCaffrey, DN Cornfield, VK Bhutani, GR Cutter, M Baier, SH Abman
}

Background. Inhaled nitric oxide improves oxygenation and lessens the need for extracorporealmembrane oxygenation in full-term neonates with hypoxaemic respiratory failure and persistent pulmonary hypertension, but potential adverse effects are intracranial haemorrhage and chronic lung $\stackrel{2}{\rightarrow}$ disease. We investigated whether low-dose inhaled nitric oxide would improve survival in premature $N$ neonates with unresponsive severe hypoxaemic respiratory failure, and would not increase the frequency 8 or severity of intracranial haemorrhage or chronic lung disease. Methods. We did a double-blind, randomised controlled trial in 12 perinatal centres that provide tertiary care. 80 premature neonates (gestational age $\leqslant 34$ weeks) with severe hypoxaemic respiratory failure were randomly assigned inhaled nitric oxide $(n=48)$ or no nitric oxide $\left(n=32\right.$, controls). Our primary outcome was survival to discharge. $\frac{\varrho}{\varnothing}$ Analysis was by intention to treat. We studied also the rate and severity of intracranial haemorrhage, $\stackrel{\varrho}{\Rightarrow}$ pulmonary haemorrhage, duration of ventilation, and chronic lung disease at 36 weeks' postconceptional $\overline{3}$ age. Findings. The two groups did not differ for baseline characteristics or severity of disease. Inhaled nitric oxide improved oxygenation after $60 \mathrm{~min}(p=0.03)$. Survival at discharge was $52 \%$ in the inhalednitric-oxide group and $47 \%$ in controls $(p=0.65)$. Causes of death were mainly related to extreme prematurity and were similar in the two groups. The two groups did not differ for adverse events or outcomes (intracranial haemorrhage grade 2-4, 28\% inhaled nitric oxide and 33\% control; pulmonary $\stackrel{\times}{\circ}$ haemorrhage $13 \%$ and $9 \%$; chronic lung disease $60 \%$ and $80 \%$ ). Interpretation. Low-dose inhaled nitric $\underline{3}$. oxide improved oxygenation but did not improve survival in severely hypoxaemic premature neonates. Low-dose nitric oxide in the most critically ill premature neonates does not increase the risk of intracranial haemorrhage, and may decrease risk of chronic lung injury. (Lancet 1999;354:1061-5)

\section{Early compared with delayed inhaled nitric oxide in moderately hypoxaemic neonates with respiratory failure: a randomised controlled trial}

\section{The Franco-Belgium Collaborative NO Trial Group}

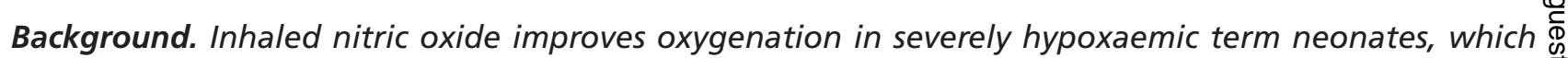
lessens the need for extracorporeal-membrane oxygenation. Improvement in other relevant outcomes: remains unknown, and safety of inhaled nitric oxide is uncertain in preterm neonates. We did a $0^{\circ}$ randomised controlled trial to assess use of inhaled nitric oxide in preterm and near-term neonates. Methods. We randomly assigned 204 preterm (<33 weeks) and near-term ( $\geqslant 33$ weeks) neonates with $\stackrel{\mathbb{Q}}{\Omega}$ oxygenation indices from 12.5 to 30.0 and 15 to 40, respectively, 10 parts per million (ppm) inhaled 2 nitric oxide $(n=105)$ or control ventilation therapy without nitric oxide $(n=99)$. The primary endpoint 8 was the oxygenation index at $2 \mathrm{~h}$. Analysis was done by intention to treat. Findings. 12 neonates were excluded, leaving 97 (45 preterm) in the nitric-oxide group and 95 (40 preterm) in the control group. $\frac{\overline{0}}{\overline{1}}$ The decline in oxygenation index at $2 \mathrm{~h}$ was greater in the nitric-oxide group than in the control group (IQR 6.2 [median 8.4] vs -2.9 [12.4], $p=0.005)$, but was significant only in near-term neonates ( $p=$ 0.03). Survivors assigned nitric oxide spent fewer days on mechanical ventilation and in the neonatal intensive-care unit, but this was also significant only in near-term neonates (6[3] vs 7[3] days, $p=0.05$, 
and 9[6] vs 12[9] days, $p=0.02$, respectively). Interpretation. Low-dose inhaled nitric oxide early in the course of neonatal respiratory failure improves oxygenation and shortens duration of mechanical ventilation and the length of stay in intensive care. Inhaled nitric oxide was not, however, significantly beneficial in preterm neonates. (Lancet 1999;354:1066-71)

Inhaled nitric oxide (NO) is an emerging treatment which has made the transition from bench to bedside in a relatively short time. This speedy introduction to clinical use relates partly to the attraction of delivering exogenously a naturally occurring and simple molecule. It is an invisible gas and can be administered directly to the site of action via the ventilator circuit, avoiding the need to deliver it systemically. There is a general perception that it should be safe. The instantaneous improvement in arterial oxygenation which is frequently observed with inhaled NO has been another important factor in its widespread clinical application.

Treatment with inhaled NO has been studied in numerous clinical trials in full term or near term infants with hypoxic respiratory failure and primary pulmonary hypertension of the newborn (PPHN) and has been evaluated in a Cochrane review ${ }^{2}$ The two introductory

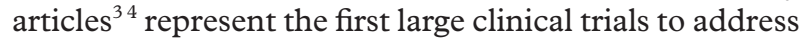
the efficacy of this agent in preterm infants with hypoxic respiratory failure. To avoid confusion with other trials discussed later in this review, the paper by Kinsella et of $p^{2}$ will be referred to as the USA trial and that by the Franco-Belgium Collaborative NO Trial Group as the Franco-Belgium trial.

The USA tria ${ }^{3}$ was a double blind, randomised, controlled trial of 80 infants of $<34$ weeks gestation with severe hypoxic respiratory failure. The intervention group received $5 \mathrm{ppm}$ inhaled $\mathrm{NO}$ and had improved oxygenation compared with controls, but there was no difference between the groups for survival. Adverse outcomes, including the development of chronic lung disease and intracranial haemorrhage, yere similar in both groups. The Franco-Belgium trial included preterm and near term infants with respiratory failure and moderate hypoxia. These infants were randomly assigned to receive either $10 \mathrm{ppm}$ inhaled NO or ventilation without NO. Oxygenation improved significantly in the near term but not the preterm infants and, again, there was no difference in mortality between the two groups.

\section{Mechanisms of action}

Inhaled $\mathrm{NO}$, or endothelium derived relaxant factor, is important in regulating vascular tone. It is generated enzymatically by nitric oxide synthase from L-arginine. NO diffuses from the vascular endothelium into the vascular smooth muscle where it activates guanylate cyclase leading to production of cyclic guanosine monophosphate. This causes the relaxation of vascular smooth muscle by a mechanism which probably involves inhibition of activation induced increase in cytosolic calcium concentration.7

It has been suggested that the improvement in oxygenation observed when inhaled $\mathrm{NO}$ is administered occurs by two main mechanisms Firstly, it may reduce pulmonary vascular resistance and thus reverse extrapulmonary shunting by selective pulmonary vasodilatation. This mechanism is likely to be important in the improved oxygenation observed in PPHN. Secondly, inhaled NO may reverse intrapulmonary shunting by redistribution of pulmonary blood flow and enhanced ventilation perfusion matching 1 11 11 This mechanism is $\frac{\overline{\bar{O}}}{\bar{n}}$ likely to be important in conditions such as parenchymal lung disease.

\section{Inhaled NO and primary pulmonary hypertension of the newborn (PPHN)}

Initial studies of the use of inhaled NQ in animal models of PPHN showed encouraging results $\mathrm{S}^{13}$ In adults with primary pulmonary hypertension inhaled NO reduced $\stackrel{x}{0}$ pulmonary vascular resistance with no effect on systemic on vascular resistance ${ }^{14}$ Not surprisingly, then, the first $\frac{\text { ? }}{0}$ uncontrolled clinical studies of $\mathrm{N}$ in newborn infants were in the group with PPHN 1 if

A Cochrane systematic reviev has summarised the of results of eight trials of inhaled $\mathrm{NO}$ treatment in term $\vec{O}$ or near term infants with hypoxaemic respiratory failure. 을 Oxygenation was found to be improved in approximately $\vec{\overrightarrow{ }}$ half of the infants treated with inhaled NO together with a reduced need for extracorporeal membrane oxy- $\frac{C}{\infty}$ genation (ECMO). Interestingly, whether infants had $\underset{N}{N}$ echocardiographic evidence of PPHN or not did not 8 appear to affect their outcomes. A weakness of most of. the included trials was that they allowed back up treatment of controls (usually with inhaled NO) who failed on the treatment to which they were randomised. They were thus unable to assess the effect of inhaled NO on survival, the need for ECMO, or other long term outcomes.

PPHN is a complex disorder characterised by marked pulmonary hypertension, right to left shunting of blood across either a patent ductus arteriosus and/or patent foramen ovale, and critical hypoxaemia. ${ }^{17}$ This condition may occur as a primary condition of neonatal maladaptation but is frequently associated with severe parenchymal lung disease such as surfactant deficiency, $\frac{0}{3}$. meconium aspiration, and bacterial pneumonia. Thus, intrapulmonary shunting may further complicate the clinical course. Because of the frequent association with parenchymal lung disease, the use of a pharmacological agent to dilate the pulmonary vasculature may not, on its own, lead to sustained improvement in oxygenation and ultimately survival.

Some trials have compared the use of inhaled NO $N$ with other ventilatory strategies. In a trial included in $\underset{\omega}{\mathrm{\omega}}$ the Cochrane reviev ${ }^{17} 205$ infants with severe PPHN were randomised to receive either inhaled NO or high 6 frequency oscillatory ventilation. The protocol allowed those who were defined as having failed on the treatment $\stackrel{?}{?}$ to which they were randomised to cross over to the 0 alternative treatment. Treatment failures after crossover $\stackrel{0}{\mathbb{D}}$ received both forms of treatment. This trial did not show

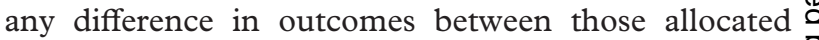
to inhaled NO and those allocated to high frequency oscillatory ventilation. In what seems to have been an $\delta$ attempt to salvage a positive result from an essentially negative trial, the investigators performed a post hoc 흘 subgroup analysis of outcome in infants after crossover $\stackrel{?}{?}$ (which was not randomised). They defined four subgroups of infants - those with respiratory distress syndrome of the newborn, meconium aspiration syndrome, idiopathic PPHN, or congenital diaphragmatic hernia. 
There was a suggestion that infants with PPHN and parenchymal lung disease who received both high frequency oscillatory ventilation and inhaled NO had improved outcomes compared with either treatment alone. Conversely, those with PPHN who did not have parenchymal lung disease were treated more successfully when they received inhaled NO either alone or in combination with high frequency oscillatory ventilation than when they received high frequency oscillatory ventilation alone.

Another clinical trial, again included in the Cochrane revieu, ${ }^{18}$ investigated 235 severely hypoxic infants of $>34$ weeks gestation who were randomised to receive inhaled NO at a concentration of $20 \mathrm{ppm}$ with $100 \%$ oxygen being administered to controls. The primary outcome was death or need for ECMO and significantly more infants in the inhaled NO group survived without ECMO. When this outcome was broken down into its two components, the difference between the two groups was the need for ECMO rather than mortality. Again, a post hoc subgroup analysis was performed to see if there were any differences according to the primary diagnosis. Unlike the trial by Kinsella et al ${ }^{17}$ they found no differences in the response to treatment according to the underlying disease. This trial did not include infants with congenital diaphragmatic hernia who were studied in a separate trial of 53 patients ${ }^{19}$ In this patient group treatment with inhaled NO did not lead to a significant improvement in oxygenation compared with controls, nor did it reduce the need for ECMO or death. Indeed, death in both groups was almost $50 \%$.

\section{Assessing clinical benefit}

Most clinical trials have evaluated the following beneficial outcomes: improved oxygenation, reduced need

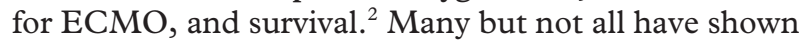
an improvement in oxygenation in the group receiving inhaled NO. Some have commented that, although treatment with inhaled NO led to an immediate apparent increase in oxygenation, this was short lived ${ }^{20}$ There is also a lack of consistency in results according to whether the inhaled NO group needed to be referred for ECMO less often than controls. However, as the criteria for ECMO referral are based on oxygenation, it is hardly surprising that some studies have referred few of the patients in the inhaled NO group, and so ECMO referral could be regarded as a proxy for oxygenation. Death in newborn infants has not been shown in any clinical trial to be reduced by treatment with inhaled NO. Survival without long term neurodevelopmental impairment is important and has been used as an outcome in trials of ECMO in neonate ${ }^{21}$ but not, so far, in clinical trials of inhaled NO. Early neurological sequelae have been evaluated and Davidson et al used what they termed a "major sequelae index" which included measures of death, ECMO, neurological sequelae in the neonatal period, and bronchopulmonary dysplasia or reactive airway disease ${ }^{22}$ There were no differences in this outcome between treatment and control groups.

\section{Assessing toxicity and adverse effects}

The adverse, or potentially adverse, outcomes which have been evaluated in newborn infants include systemic hypotension, measurements of potentially damaging products of inhaled NO, chronic lung disease of prematurity, methaemoglobinaemia, and intracranial haemorrhage. Systemic hypotension has not been described in any of the clinical trials in neonates. 123 Very little direct toxicity of inhaled NO has been noted and discussion of the possible mechanisms by which inhaled NO may cause toxicity or, indeed, may be protective to the lungs, is largely speculative.

Nitric oxide is rapidly oxidised in air to nitrogen dioxide which has been implicated in airway damage and $\overrightarrow{\vec{m}}$ inflammation ${ }^{24}$ The concentration of nitrogen dioxide is $\overrightarrow{0}$ proportional to the second power of inspired $\mathrm{NO}$ and $\frac{\bar{\sigma}}{\sigma}$ the first power of inspired oxygen, so inspired nitrogen $\overline{\bar{p}}$ dioxide is likely to be increased when the concentration $\frac{\mathbb{Q}}{2}$ of both inhaled NQ and the concentration of inspired 0 oxygen is increased ${ }^{22}$ It has been shown that the use of $\omega$ inhaled NO in a neonatal ventilator with an $\mathrm{FiO}_{2}$ of 0.9 ? can prodyce $5 \mathrm{ppm}$ nitrogen dioxide in less than $20 \overrightarrow{\vec{\omega}}$ seconds. ${ }^{25}$

There are also concerns that nitric oxide itself is a $\overrightarrow{\overrightarrow{0}}$ free radical and is converted to peroxypitrite which may $\underset{\nabla}{2}$ result in membrane lipid peroxidation ${ }^{26}$ There is some $x$ evidence that, at limited concentrations, peroxynitrite of may protect thelyngs, but at high concentrations leads $\frac{\text { D }}{0}$ to lung damage. ${ }^{27}$ Free radical damage may cause surfactant dysfunction and lead to chronic lung disease ${ }^{2}$. These mechanisms may be particularly pertinent if this of treatment is extended to preterm infants who, unlike $\overrightarrow{0}$ term infants, have free iron in the plasma which may $\stackrel{0}{?}$ add to the risk of oxygen damage induced by inhaled $\mathrm{NO}{ }^{24}$

If inhaled NO causes direct lung toxicity by any of $\frac{6}{\infty}$ these mechanisms, it may be associated with an increase $\stackrel{\vec{N}}{N}$ in chronic lung disease. Because of the difficulty in 8 assessing long term outcomes in these studies, and because of problems with trial designs, this has not yet been properly evaluated. A recently published large trial of inhaled NO in PPHN, which is too recent to have been included in the Cochrane review, showed that inhaled NO reduced the need for ECMO and the $\stackrel{\mathbb{Q}}{2}$ incidence of chronic lung disease. ${ }^{29}$ However, in this $\overrightarrow{\overrightarrow{0}}$ group of infants of $>34$ weeks gestation chronic lung 3 disease was defined as need for supplemental oxygen at 30 days and the number of infants receiving oxygen after discharge was similar in the two groups.

High doses of inhaled NO have been associated with a prolonged bleeding tim $\$^{30}$ which has raised concerns about the risk of intracranial haemorrhage. In preterm 3 infants laboratory based measures of bleeding tendency are imprecise and variable and have not been evaluated 3 in many clinical trials. The USA trial performed a 0 very careful evaluation of the infants for intracranial haemorrhage and, in fact, it calculated the risk of intra- $\frac{7}{2}$ cranial haemorrhage based on an assumption that all those who were treated with inhaled NO and who died $N$ before the age of seven days with unknown intracranial $N$ haemorrhage status actually had grade 4 intracranial $\underset{\omega}{N}$ haemorrhage. Even with this "worse case scenario" they showed no increase in risk of intracranial haemorrhage 0 in the inhaled NO group, which is very reassuring. A trial that is adequately powered to measure long term ? adverse outcomes is likely to be very large. The USA 0 trial estimated that a clinical trial designed to show $\frac{\vec{D}}{\mathbb{D}}$ a significant increase in risk for grade 4 intracranial haemorrhage would require 15000 neonates, although the assumptions on which this calculation was based were not stated.

\section{Appropriate dose of inhaled NO}

Over the last decade, since clinical trials have been performed with this agent, the dose used has reduced considerably, ranging from $80 \mathrm{ppm}$ to $2 \mathrm{ppm}$. This has been largely mitigated by concerns about toxicity. At a 
dose of $80 \mathrm{ppm}$ and occasionally at lower doses, ${ }^{20}$ increased conceptrations of methaemoglobin NO have been observed. $\left.{ }^{2}\right|^{3}$ Davidson et af $2^{2}$ randomised infants with PPHN into four groups receiving $0 \mathrm{ppm}$ (control), 5,20 or $80 \mathrm{ppm}$ inhaled $\mathrm{NO}$ and found no difference in efficacy between the doses. Cornfield et a $\beta^{2}$ compared inhaled $\mathrm{NO}$ in a dose of $2 \mathrm{ppm}$ with a control group who did not receive inhaled NO. Treatment failures after one hour in either group were treated with inhaled $\mathrm{NO}$ at $20 \mathrm{ppm}$. Infants given the $2 \mathrm{ppm}$ dose had a slower rate of clinical deterioration than controls. The $2 \mathrm{ppm}$ dose was found not to improve oxygenation whereas the $20 \mathrm{ppm}$ dose did. Infants who were initially given $2 \mathrm{ppm}$ did not show an improvement in oxygenation when $20 \mathrm{ppm}$ inhaled NO was used but those in the control group did. This has raised the possibility that administration of a subtherapeutic dose of inhaled NO may attenuate the response to endothelium derived vasodilator agents such as endogenous nitric oxide or prostacyclin. It would appear that the minimum dose that has been shown to be effective in improving oxygenation in clinical trials in neonates is around $5 \mathrm{ppm}$ and, in a study of preterm infants, the $5 \mathrm{ppm}$ dose was shown to be of equivalent efficacy to $20 \mathrm{ppm}$

\section{Inhaled NO in preterm infants}

Given the lack of convincing benefits associated with NO treatment in term and near term infants, there are understandable concerns about extending these investigations to small preterm infants. Until the publication of the USA and Franco-Belgium trials, only one small clinical trial by Subhedar et al had compared the use of inhaled NO in preterm infants with a group of infants not given inhaled NO. ${ }^{34}$ This was the only trial suitable for inclusion in a Cochrane review of inhaled NO for preterm infants with respiratory failure. ${ }^{23}$ It investigated the effect of inhaled $\mathrm{NO}$ and intravenous dexamethasone on death or development of chronic lung disease in 42 infants deemed to be at high risk of developing chronic lung disease. No difference in outcome was found for either treatment.

In preterm infants with respiratory distress syndrome of the newborn, hypoxaemia is predominately the result of intrapulmonary shunting and poor ventilation perfusion matching. Although right to left extrapulmonary shunting may contribute to this hypoxaemia, Subhedar et al found that none of the preterm infants included in their trial had Doppler evidence of significant extra-

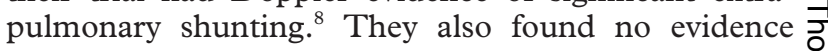
of a higher pulmonary artery pressure in infants who responded (by improved oxygenation) to inhaled NO compared with those who did not respond. This is $\overrightarrow{\vec{F}}$ different from the findings in studies of term or near $\overrightarrow{0}$ term infants where a high proportion had an intracardiac $\frac{C}{0}$ shunt which was either right to left or bidirectional. ${ }^{1 / 2} 20$ Subhedar et al showed that inhaled NO was associated $\frac{\mathbb{Q}}{\varnothing}$ with a fall in pulmonary artery pressure while mean systemic arterial pressure was maintained. The oxygenation response was weakly associated with an in- $\overrightarrow{0}$ crease in pulmonary blood flow but not with the $\overrightarrow{\vec{\omega}}$ reduction in pulmonary artery pressure. These results $\stackrel{\sigma}{\sigma}$ may be difficult to interpret as a global increase in $\overrightarrow{\overrightarrow{0}}$ pulmonary blood flow without redistribution of pulmonary perfusion should lead to further impairment of $\dot{x}$ gas exchange by worsening the ventilation perfusion of mismatch or the intrapulmonary shunting. This is likely to occur when systemic vasodilators are administered. 3 음 One attraction of inhaled NO is that it may be selectively $\overrightarrow{.}$ deposited only in those regions of the lungs which are is ventilated, leading to a local relaxation in the tone of vessels surrounding ventilated areas and simultaneously $\stackrel{\circ}{?}$ causing no change in the tone of vessels surrounding $\vec{\longrightarrow}$ non-ventilated areas. The net effect yould lead to an overall improvement is gas exchange. ${ }^{10}$

\section{The future}

Those investigating inhaled $\mathrm{NO}$ in clinical trials of preterm infants can learn much from trials conducted in bigger neonates. In order to evaluate outcomes properly, $\overline{0}$ crossover to inhaled NO should be avoided and it was disappointing that this occurred in the Franco-Belgium $\stackrel{\mathbb{2}}{\varrho}$ trial. Blinding is possible with inhaled $\mathrm{NO}$ and should $\overrightarrow{\overrightarrow{0}}$ be used, as was the case for the USA trial but not the 3 Franco-Belgium trial. Long term follow up to assess neurodevelopmental outcomes and chronic lung disease is essential and this is planned for the USA trial. Consideration of subgroups is important, but the trials should be designed to investigate the efficacy of treatment in individual subgroups and post hoc subgroup analysis should be avoided. Respiratory distress syndrome of the newborn is the underlying diagnosis in 3 most preterm infants, but severity varies. Most of the

\section{LEARNING POINTS}

* Inhaled nitric oxide is an emerging treatment which may be of benefit in patients with conditions causing severe hypoxia

* Inhaled nitric oxide in term or near term infants with hypoxic respiratory failure has $\stackrel{\oplus}{\rightarrow}$ improved oxygenation but trials have been unable to assess its effect on survival or long term outcomes

* The two studies described are the first large clinical trials to address the efficacy of inhaled $\stackrel{\circ}{\stackrel{\circ}{\circ}}$ nitric oxide in preterm infants with hypoxic respiratory failure

* In one study the inhaled nitric oxide group had improved oxygenation compared with controls, but there was no difference in groups for survival, occurrence of chronic lung disease, or intracranial haemorrhage

* There is still no evidence that inhaled nitric oxide in preterm infants improves survival or important long term outcomes such as chronic lung disease or neurodevelopmental status. Further clinical trials are needed to evaluate these outcomes 
clinical trials have been in severely hypoxic infants, but it will be important in future trials to investigate whether a beneficial effect is observed in groups with different degrees of severity.

The use of inhaled NO in patients with hypoxia is proliferating. Recent clinical trials which have included childre ${ }^{36}$ and adult $\beta^{37}$ with severe respiratory distress syndrome have failed to show an improvement in clinically important outcomes. This treatment is now being used, in the absence of evidence of benefit, in infants and children on intensive care units.

One of the most interesting aspects of this whole field is to consider how new or emerging treatments should be evaluated in extremely sick and vulnerable newborn infants. It has been suggested that any new treatment should be applied in a randomised manner from the first patient ${ }^{38}$ This approach, however, takes no account of the total number needed to evaluate clinically important outcomes. Many investigators have alluded to the large number of centres that are using inhaled NO, both inside and outside clinical trial protocols, and this has led to considerable problems with recruitment to clinical trials ${ }^{22}$ Most clinical trials are underpowered and were stopped early, frequently because of poor recruitment. Many were single rather than multicentre.

The usual approach in the evolution of new treatments from bench to bedside is firstly to undertake studies in appropriate animal models. Even if the results of animal studies are encouraging, the decision about when to move to well planned clinical studies of a new agent may be difficult and these are generally first conducted in adults. ${ }^{30}$ Assuming that the results continue to show safety and efficacy, these may be followed by early explanatory trial ${ }^{40}$ before large, pragmatic clinical trials are finally conducted. Not until the last group of studies has been completed and shown unequivocal benefit without unacceptable adverse effects should the treatment be used outside the context of a clinical trial.

This process has been undertaken very successfully in newborn infants with treatments such as surfactant administration for neonatal lung disease. Although both the USA and the Franco-Belgium trials have added important new information, inhaled NO must still be regarded as an experimental treatment for preterm infants with hypoxic respiratory failure. Unless further clinical trials are conducted, answers to important questions - particularly concerning long term outcomes of these treatments - will not be provided. In the meantime, the only real conclusion that can be drawn from these and other studies is that inhaled NO in preterm infants should only be used in the context of a clinical trial.

1 Day R, Lynch J, White K, et al. Acute response to inhaled nitric oxide in newborns with respiratory failure and pulmonary hypertension. Pediatrics 1996;98:698-705.

2 Finer N, Barrington K. Nitric oxide for respiratory failure in infants born at or near term (Cochrane Review). Oxford: Update Software, 2000.

3 Kinsella J, Walsh W, Bose C, et al. Inhaled nitric oxide in premature neonates with severe hypoxaemic respiratory failure: a randomised controlled trial. Lancet 1999;354:1061-5.

4 The Franco-Belgium Collaborative NO Trial Group. Early compared with delayed inhaled nitric oxide in moderately hypoxaemic neonates with respiratory failure: a randomised controlled trial. Lancet 1999; with respiratory

5 Palmer R, Ferrige A, Moncada S. Nitric oxide release accounts for the biological activity of endothelium-derived relaxing factor. Nature 1987; 327:524-6.
6 Palmer R, Ashton D, Moncada S. Vascular endothelial cells synthesize nitric oxide from L-arginine. Nature 1988;333:664-6.

7 Ignarro L. Biological actions and properties of endothelium-derived nitric oxide formed and released from artery and vein. Circ Res 1989,

8 Subhedar N, Shaw N. Changes in oxygenation and pulmonary haemodynamics in preterm infants treated with inhaled nitric oxide. Arch Dis Child 1997;77:F191-7.

9 Frostell C, Fratacci M, Wain J, et al. Inhaled nitric oxide: a selective pulmonary vasodilator reversing hypoxic pulmonary vasoconstriction. Circulation 1991;83:2038-47.

10 Roze J, Storme L, Zupan V, et al. Echocardiographic investigation of inhaled nitric oxide in newborn babies with severe hypoxaemia. Lancet 1994;344:303-5.

11 Rossaint R, Falke K, Lopez F, et al. Inhaled nitric oxide for the adult respiratory distress syndrome. N Engl f Med 1993;328:399-405.

12 Zayek M, Cleveland D, Morin F. Treatment of persistent pulmonar hypertension in the newborn lamb by inhaled nitric oxide. 7 Pediatr 1993;122:743-50

13 Zayek M, Wild L, Roberts J, et al. Effect of nitric oxide on the survival rate and incidence of lung injury in newborn lambs with persistent pulmonary hypertension. $\mathcal{F}$ Pediatr 1993;123:947-52.

14 Pepke-Zaba J, Higenbottam T, Dinh-Xuan A, et al. Inhaled nitric oxide as a cause of selective pulmonary vasodilatation in pulmonary hypertension. Lancet 1991;338:1173-4.

15 Roberts J, Polaner D, Lang P, et al. Inhaled nitric oxide in persistent pulmonary hypertension of the newborn. Lancet 1992;340:818-9.

16 Kinsella J, Neish S, Shaffer E, et al. Low-dose inhalational nitric oxide of in persistent pulmonary hypertension of the newborn. Lancet 1992; is 340:819-20.

17 Kinsella J, Truog W, Walsh W, et al. Randomised, multicenter trial of $\frac{\overline{0}}{0}$ inhaled nitric oxide and high-frequency oscillatory ventilation in severe, persistent pulmonary hypertension of the newborn. F Pediatr 1997;131:55-62.

18 The Neonatal Inhaled Nitric Oxide Study Group (NINOS). Inhaled $\mathcal{C}$ nitric oxide in full-term and nearly full-term infants with hypoxic respiratory failure. N Engl F Med 1997;336:597-604

19 The Neonatal Inhaled Nitric Oxide Study Group (NINOS). Inhaled 윽 nitric oxide and hypoxic respiratory failure in infants with congenital diaphragmatic hernia. Pediatrics 1997;99:838-45.

20 Barefield E, Karle V, Philips J, et al. Inhaled nitric oxide in term infants with hypoxemic respiratory failure. F Pediatr 1996;129:297-86.

21 UK Collaborative ECMO Group. UK collaborative randomised trial o of neonatal extracorporeal membrane oxygenation. Lancet 1996;348: $75-82$.

22 Davidson D, Barefield E, Kattwinkel J, et al. Inhaled nitric oxide for the early treatment of persistent pulmonary hypertension of the term newborn: a randomised, double-masked, placebo-controlled, doseresponse, multicenter study. Pediatrics 1998;101:325-34.

23 Barrington K, Finer N. Inhaled nitric oxide for respiratory failure in preterm infants (Cochrane Review). Oxford: Update Software, 2000

24 Saugstad O. Inhaled nitric oxide for preterm infants: still an experimental therapy. Lancet 1999; 354:1047-8.

25 Bouchet M, Renaudin M, Raveau C, et al. Safety requirement for the use of inhaled nitric oxide in neonates. Lancet 1993;341:968-9.

26 Radi R, Beckman J, Bush K, et al. Peroxynitrite-induced membrane lipid peroxidation. Arch Biochem Biophys 1991;288:481-7.

27 Gaston B, Drazen J, Loscalzo J, et al. The biology of nitrogen oxides in the airways. Am f Respir Crit Care Med 1994;149:538-51.

28 Haddad I, Ischiropoulos H, Holm B, et al. Mechanisms of peroxynitriteinduced injury to pulmonary surfactants. Am f Physiol 1993;265: L555-64.

29 Clark R, Kueser T, Walker M, et al. Low-dose nitric oxide therapy for persistent pulmonary hypertension of the newborn. $N$ Engl f Med 2000;342:469-74.

30 Hogman M, Frostell C, Arnberg $\mathrm{H}$, et al. Bleeding time prolongation and NO inhalation. Lancet 1993:341:1664-5.

31 Roberts J, Fineman J, Frederick C, et al. Inhaled nitric oxide and persistent pulmonary hypertension of the newborn. $N$ Engl $7 \mathrm{Med}$ persistent pulmonar

32 Cornfield D, Maynard R, O deRegnier R, et al. Randomized, controlled trial of low-dose inhaled nitric oxide in the treatment of term and $\subseteq$ near-term infants with respiratory failure and pulmonary hypertension. Pediatrics 1999;104:1089-94

33 Skimming J, Bender K, Hutchison A, et al. Nitric oxide inhalation in infants with respiratory distress syndrome. F Pediatr 1997;130:225-30.

34 Subhedar N, Ryan S, Shaw N. Open randomised controlled trial of $\mathrm{N}$ inhaled nitric oxide and early dexamethasone in high risk preterm infants. Arch Dis Child 1997;77:F185-90.

35 Radermacher P, Santak P, Becker H, et al. Prostaglandin E1 and $\mathrm{N}$ nitroglycerin reduce pulmonary capillary wedge pressure but worsen $\omega$ $\mathrm{V} / \mathrm{Q}$ distribution in patients with adult respiratory distress syndrome. $\mathbf{O}$ Anesthesiology 1989;70:601-9.

36 Dobyns E, Cornfield D, Anas N, et al. Multicenter randomized con- $\boldsymbol{C}$ trolled trial of the effects of inhaled nitric oxide therapy on gas $\frac{\bar{D}}{\mathrm{D}}$ exchange in children with acute hypoxemic respiratory failure. F Pediatr $\&$ 1999;134:406-12.

37 Michael J, Barton R, Saffle J, et al. Inhaled nitric oxide versus con- $T$ ventional therapy: effect on oxygenation in ARDS. Am f Respir Crit Care Med 1998;157:1372-80.

38 Chalmers T. Randomization of the first patient. Med Clin North Am 1975;59:1035-8.

39 Smyth RL, Weindling AM. Research in children: ethical and scientific aspects. Lancet 1999;354(Suppl 2):SII21-4.

40 Smyth RL Evidence-based pediatric pulmonary medicine: how can it help? (see comments). Pediatr Pulmonol 1998;25:118-27.

$\frac{1}{20}$

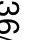

,

.

$\overrightarrow{0}$

(1)

